\title{
Diffusion-driven codimension-2 Turing-Hopf bifurcation in general Brusselator model
}

\author{
Lei Kong ${ }^{1}$ and Changrong $\mathrm{Zhu}^{2}$ \\ ${ }^{1}$ Guizhou University of Finance and Economics \\ ${ }^{2}$ Chongqing University
}

May 11, 2020

\begin{abstract}
The spatiotemporal dynamics for general reaction-diffusion systems of Brusselator type under the homogeneous Neumann boundary condition is considered. It is shown that the reaction-diffusion system has a unique steady state solution. For some suitable ranges of the parameters, we prove that the steady state solution can be a codimension-2 Turing-Hopf point. To understand the spatiotemporal dynamics in the vicinity of the Turing-Hopf bifurcation point, we calculate and analyze the normal form on the center manifold by analytical methods. A wealth of complex spatiotemporal dynamics near the degenerate point are obtained. It is proved that the system undergoes a codimension-2 Turing-Hopf bifurcation. Moreover, several numerical simulations are carried out to illustrate the validity of our theoretical results.
\end{abstract}

\section{Hosted file}

lunwen4-20181203.pdf available at https://authorea.com/users/320245/articles/449839diffusion-driven-codimension-2-turing-hopf-bifurcation-in-general-brusselator-model 
figures/parameter-cure5/parameter-cure5-eps-converted-to.pdf 
figures/E0-1/E0-1-eps-converted-to.pdf 
figures/E02/E02-eps-converted-to.pdf 
figures/E012-1/E012-1-eps-converted-to.pdf 
figures/E0123/E0123-eps-converted-to.pdf 
figures/E012-2/E012-2-eps-converted-to.pdf 
figures/E01/E01-eps-converted-to.pdf 